\title{
(2) OPEN ACCESS \\ Accuracy of PE rule-out strategies in pregnancy: secondary analysis of the DiPEP study prospective cohort
}

\author{
Steve Goodacre (1) , ${ }^{1}$ Catherine Nelson-Piercy, ${ }^{2}$ Beverley J Hunt, ${ }_{1}^{3}$ Gordon Fuller (1)
}

${ }^{1}$ School of Health and Related Research, University of Sheffield, Sheffield, UK

${ }^{2}$ Women's Health Academic Centre, Guy's \& St Thomas's NHS Foundation Trust, London, UK

${ }^{3}$ Departments of Haematology and Rheumatology, Guy's \& St Thomas's NHS Foundation Trust, London, UK

\section{Correspondence to} Professor Steve Goodacre, School of Health and Related Research, University of Sheffield, Sheffield, S1 4DA, UK; s.goodacre@sheffield.ac.uk

Received 17 October 2019 Revised 20 February 2020 Accepted 3 March 2020 Published Online First 9 April 2020
ABSTRACT

Objective Recent studies suggest that combinations of clinical probability assessment (the YEARS algorithm or Geneva score) and D-dimer can safely rule out suspected pulmonary embolism (PE) in pregnant women. We performed a secondary analysis of the DiPEP (Diagnosis of Pulmonary Embolism in Pregnancy) study data to determine the diagnostic accuracy of these strategies. Methods The DiPEP study prospectively recruited and collected data and blood samples from pregnant/ postpartum women with suspected PE across 11 hospitals and retrospectively collected data from pregnant/postpartum women with diagnosed PE across all UK hospitals (15 February 2015 to 31 August 2016). We selected prospectively recruited pregnant women who had definitive diagnostic imaging for this analysis. We used clinical data and D-dimer results to determine whether the rule out strategies would recommend further investigation. Two independent adjudicators used data from imaging reports, treatments and adverse events up to 30 days to determine the reference standard.

Results PEs were diagnosed in 12/219 (5.5\%) women. The YEARS/D-dimer strategy would have ruled out PE in $96 / 219(43.8 \%)$ but this would have included 5 of the 12 with PEs. Sensitivity for PE was $58.3 \%$ (95\% Cl $28.6 \%$ to $83.5 \%$ ) and specificity $44.0 \%$ (37.1\% to $51.0 \%)$. The Geneva/D-dimer strategy would have ruled out PE in $46 / 219$ (21.0\%) but this would have included three of the 12 with PE. Sensitivity was $75.0 \%(95 \% \mathrm{Cl}$ $42.8 \%$ to $93.3 \%$ ) and specificity $20.8 \%$ (95\% Cl $15.6 \%$ to $27.1 \%$ ). Administration of anticoagulants prior to blood sampling may have reduced D-dimer sensitivity for small PE.

Conclusion Strategies using clinical probability and D-dimer have limited diagnostic accuracy and do not accurately rule out all PE in pregnancy. It is uncertain whether PE missed by these strategies lead to clinically important consequences.

\section{INTRODUCTION}

Recent studies suggest that combinations of clinical probability assessment and D-dimer can safely rule out pulmonary embolism (PE) in a proportion of pregnant women presenting with suspected PE who would otherwise require imaging to rule out PE. van der Pol et $a l^{1}$ tested a pregnancy-modified YEARS algorithm and D-dimer in 498 pregnant women with suspected PE presenting to 18 hospitals over 5 years (20 with PE), ruling out PE without scan or adverse outcome in 195 (39\%). Righini et al ${ }^{2}$ tested clinical probability scoring with the Geneva score
Key messages

What is already known on this subject

- PE is an important potential cause of morbidity in pregnancy but symptoms suggesting PE are common in pregnancy.

- Recent studies suggest that a combination of clinical probability assessment and D-dimer measurement can allow safe discharge without imaging in a proportion of pregnant women with suspected PE.

\section{What this study adds}

- This secondary analysis of the DiPEP study showed that strategies using clinical probability and D-dimer have limited diagnostic accuracy and do not accurately rule out all PE in pregnancy. It is uncertain whether PE missed by these strategies lead to clinically important consequences.

alongside D-dimer in 395 pregnant women with suspected PE presenting to eleven hospitals over 8 years (28 with PE), ruling out PE without scan or adverse outcome in 46 (12\%).

These studies suggest that a proportion of women can avoid imaging for PE but may lack statistical power to ensure an acceptably low rate of adverse outcome in this group. The studies were designed to detect 3 -month event rates of $2.7 \%$ and $3.0 \%$, respectively, but it is unclear whether patients and clinicians consider these event rates acceptable for a potentially catastrophic outcome. We are not aware of any studies estimating clinician or patient willingness to accept risk of adverse outcome after discharge for suspected $\mathrm{PE}$ in pregnancy but a survey of emergency physicians reported that only $18 / 1023(1.8 \%)$ considered an adverse event rate above $2 \%$ acceptable following negative assessment for acute chest pain. ${ }^{3}$ Furthermore, the sample size was estimated and event rates reported across the entire cohort, including those who received imaging, whereas the relevant population is those who did not receive imaging. The upper 95\% CI for the zero event rates in the non-imaged population are $2.4 \%$ for the 195 women in the van der Pol study and $9.6 \%$ in the 46 women in the Righini study.

The DiPEP (Diagnosis of Pulmonary Embolism in Pregnancy) study prospectively recruited and collected data and blood samples from pregnant 
and postpartum women with suspected PE across 11 sites, and retrospectively collected data from pregnant and postpartum women with diagnosed PE across the whole of the UK. ${ }^{45}$ Clinical probability assessment and D-dimer were not routinely used to select women for investigation in the study and the relevant guidelines recommended against this practice. ${ }^{6}$ The DiPEP data, therefore, offered the opportunity to determine the diagnostic accuracy of rule out strategies, compared with a reference standard based on radiological imaging.

We aimed to undertake a secondary analysis of data and blood samples from pregnant women with suspected PE who were prospectively recruited to the DiPEP study, to determine the accuracy of rule out strategies based on clinical probability assessment and D-dimer.

\section{METHODS}

DiPEP was a prospective cohort study augmented with additional retrospective cases to determine whether clinical features, individually or in the form of a clinical decision rule, or D-dimer could be used to select pregnant and postpartum women for diagnostic imaging. ${ }^{4}$ It also involved analysis of blood samples collected from the prospective cohort to determine the accuracy of a range of biomarkers for PE in pregnancy and post partum. ${ }^{5}$

Prospectively recruited pregnant women with suspected PE were selected for this analysis. Women were identified at emergency departments and maternity units across eleven sites over 18 months and asked to provide written informed consent to data and blood sample collection. A research nurse or midwife then completed a case report form, including details of patient characteristics, investigations and treatments for venous thromboembolism (VTE). Hospital records were reviewed at 30 days after recruitment and any subsequent VTE-related investigations, treatments or adverse events were recorded. All management was at the discretion of the attending clinician and determined on the basis of patient need.

We excluded retrospectively identified women with diagnosed PE from this analysis because blood samples could not be collected from these women; and to prevent selection bias from incomplete retrospective case ascertainment, and avoid information bias from retrospective data abstraction from case notes. Results of hospital D-dimer testing were available for some but different assays and thresholds for positivity were used in each hospital. Prospectively recruited postpartum women were excluded because neither of the rule out strategies had been developed or evaluated for postpartum women.

The pregnancy-adapted YEARS algorithm used three criteria to select women for D-dimer testing: clinical signs of deep vein thrombosis (DVT), haemoptysis and PE as the most likely diagnosis. The DiPEP case report form recorded whether haemoptysis was a presenting symptom, whether there were clinical signs of DVT and what was considered the most likely diagnosis after clinical assessment. These data were used to determine the results of applying the three criteria to DiPEP patients.

Righini et $a l^{2}$ used the revised Geneva score (low or intermediate pretest clinical probability) to select women for D-dimer testing. The DiPEP case report form recorded age, heart rate, whether haemoptysis was a presenting symptom any previous history of VTE, surgery or significant injury in the previous 4 weeks, pre-existing cancer and clinical signs of DVT. A freetext box for recording any other symptoms was searched to identify patients presenting with unilateral lower limb pain. These data were used to determine the results of applying the revised Geneva score to DiPEP patients. We have previously evaluated a pregnancy-modified version of the revised Geneva score ${ }^{4}$ but for this analysis used an unmodified version to ensure consistency with the study of Righini et al. ${ }^{2}$

Serum and citrate blood samples were collected by a member of the clinical team or research nurse/midwife using good venepuncture technique, ideally while obtaining routine blood samples for standard clinical assessment in diagnostic workup. The samples were centrifuged at $2000 \mathrm{~g}$ for $15 \mathrm{~min}$ at room temperature and frozen down within 4 hours of being obtained. Citrate samples were further processed to obtain platelet free plasma.

Plasma and serum samples were stored in aliquots labelled with the patient ID and the storage box coordinates recorded on paper and electronic study documentation, according to local protocols. The samples were stored in $-70^{\circ} \mathrm{C}$ freezers at each participating hospital (with the exception of one location where $\mathrm{a}-40^{\circ} \mathrm{C}$ freezer was used) for the duration on the study until all samples were transported for analysis to Guy's St Thomas Trust, London, UK.

The Zymutest D-Dimer Eliza assay (Quadratech Diagnostics, Epsom, UK) was used to measure the D-Dimers by ELISA according to the manufacturer's instructions. The recommended threshold for the test was $400 \mathrm{ng} / \mathrm{mL}$. The coefficient of variation was $4.6 \%$ intra-assay and $10.8 \%$ inter assay for the Zymutest D-Dimer. In accordance with the strategy of Righini et al, we used the recommended threshold of $400 \mathrm{ng} / \mathrm{mL}$ to rule out PE in women with a low or intermediate revised Geneva score. In accordance with the diagnostic strategy tested by van der Pol et $a l$, we used the recommended threshold for positivity of $400 \mathrm{ng} /$ $\mathrm{mL}$ to rule out PE in women when one or more of the YEARS criteria and $800 \mathrm{ng} / \mathrm{mL}$ (double the recommended threshold for positivity) when none of the YEARS criteria were met.

The reference standard was determined by two independent observers, who reviewed reports of VTE-related diagnostic imaging, treatments and adverse events up to 30 days after recruitment. Any disagreements were settled by a third independent expert. The reviewers were blinded to index test results.

In accordance with the primary analysis plan for DiPEP, this analysis was limited to those with conclusive imaging. This meant that the reference standard was effectively based on imaging results alone. A secondary analysis explored the effect of including those with inconclusive or no imaging. Analysis was descriptive, involving estimation of the sensitivity and specificity of each strategy for diagnosing PE, with a 95\% CI.

\section{Patient and public involvement}

The DiPEP study steering committee included representatives from two patient organisations, Thombosis UK and the Sheffield Emergency Care Forum. They advised on the design, conduct and interpretation of the study, and assisted with dissemination of the findings.

\section{RESULTS}

A total of 324 women were recruited across 11 participating sites between 15 February 2015 and 31 August 2016. We excluded 55 who were post partum and a further 9 who did not have D-dimer measurements recorded. The analysis was limited to 219 women with conclusive imaging (five were excluded with clinically diagnosed PE and 36 with clinically ruled out PE). Figure 1 shows the flow of participants through the study and table 1 outlines the characteristics of those included in the analysis.

PE was diagnosed in 12/219 (5.5\%). Table 2 shows the maternal and gestational age for each woman with PE and how 


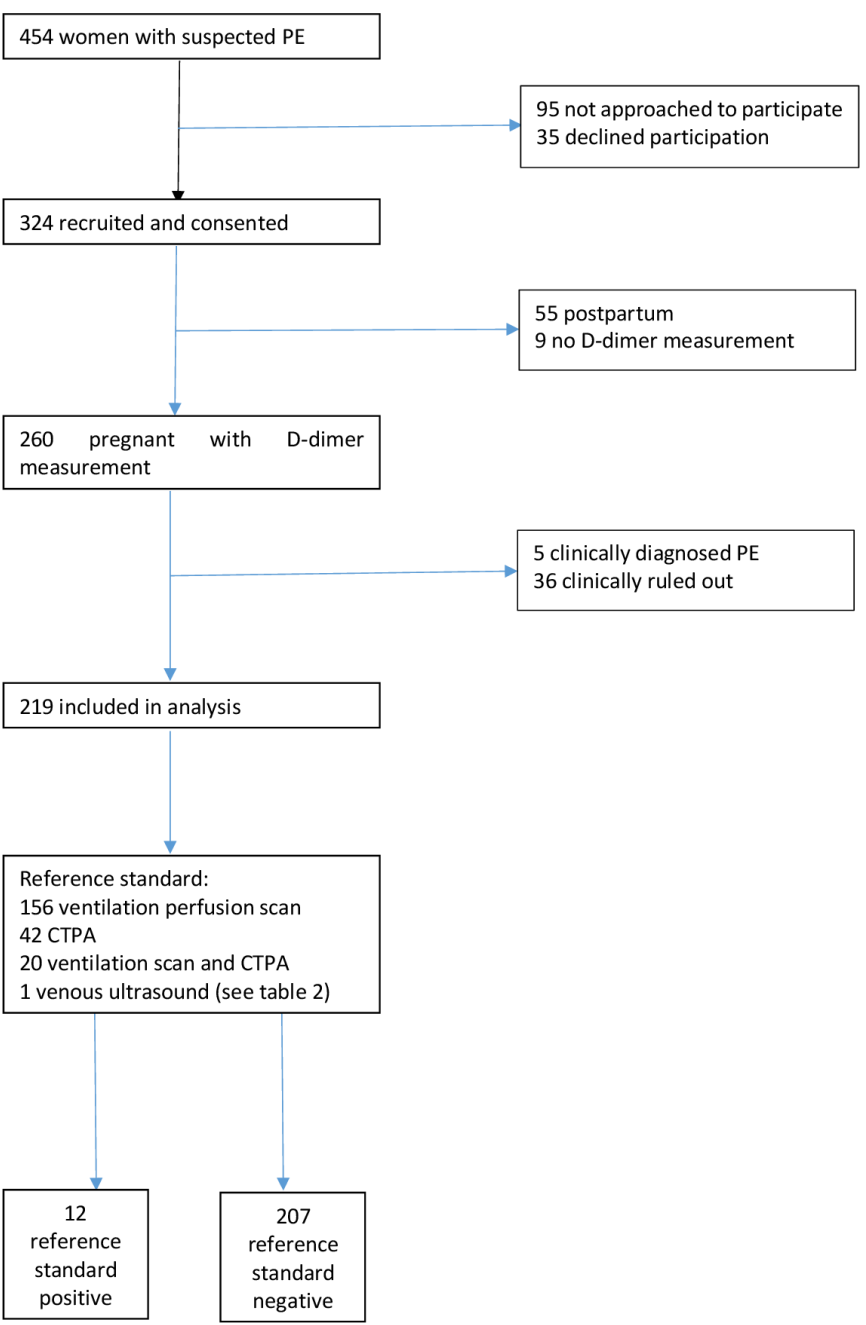

Figure 1 Flow of participants through the study. PE, pulmonary embolism; CTPA, computed tomography pulmonary angiogram

PE was diagnosed on imaging. The diagnosis was based on computed tomography pulmonary angiogram (CTPA) findings for five women, ventilation-perfusion scanning for six women, and lower limb ultrasound evidence of DVT in the presence of symptoms of PE for one woman.

Table 3 shows the results of applying the YEARS/D-dimer (van der Pol) strategy to this cohort and table 4 shows the results of applying Geneva/D-dimer (Righini) strategy. The YEARS/D-dimer strategy would have resulted in 96/219 women (43.8\%) being discharged without imaging, but this would have included 5/12 with PE. The sensitivity of the strategy was 58.3\% (95\% CI $28.6 \%$ to $83.5 \%)$ and specificity was $44.0 \%$ (37.1\% to $51.0 \%)$. The Geneva/D-dimer strategy would have resulted in $46 / 219$ women $(21.0 \%)$ being discharged without scanning, but this would have included $3 / 12$ with PE. The sensitivity of the strategy was $75.0 \%$ (42.8\% to $93.3 \%)$ and specificity was $20.8 \%(15.6 \%$ to $27.1 \%)$.

Secondary analysis including those with inconclusive or no imaging produced similar results. The YEARS/D-dimer strategy sensitivity was $52.9 \%(28.5 \%$ to $76.1 \%)$ and specificity $42.4 \%$ (36.1\% to $48.9 \%)$. The Geneva/D-dimer strategy sensitivity was $70.6 \%(44.1 \%$ to $88.6 \%)$ and specificity $19.8 \%(15.1 \%$ to $25.4 \%)$.

Table 5 shows the elements of each strategy and whether the overall strategy indicated PE (requiring imaging) or no PE (no
Table 1 Characteristics of the study population

\begin{tabular}{|c|c|}
\hline Characteristic & Patients $(n=219)$ \\
\hline Mean age & 29.3 years \\
\hline Mean BMI & $27.9 \mathrm{~kg} / \mathrm{m}^{2}$ \\
\hline Mean heart rate & $97.8 / \mathrm{min}$ \\
\hline Mean respiratory rate & $18.9 / \mathrm{min}$ \\
\hline Mean oxygen saturation & $97.8 \%$ \\
\hline Mean systolic blood pressure & $120.7 \mathrm{~mm} \mathrm{Hg}$ \\
\hline Mean diastolic blood pressure & $71.9 \mathrm{~mm} \mathrm{Hg}$ \\
\hline Mean temperature & $36.5^{\circ} \mathrm{C}$ \\
\hline \multicolumn{2}{|l|}{ Smoking status } \\
\hline Never & $150(68.5 \%)$ \\
\hline Gave up before & $28(12.8 \%)$ \\
\hline Gave up during & $15(6.8 \%)$ \\
\hline Current & $26(11.9 \%)$ \\
\hline One or more previous pregnancies $<24$ weeks & $86(39.3 \%)$ \\
\hline One or more previous pregnancy $>24$ weeks & $137(62.6 \%)$ \\
\hline Family history of thrombosis & $41(18.7 \%)$ \\
\hline History of varicose veins & $18(8.2 \%)$ \\
\hline History of intravenous drug use & $1(0.5 \%)$ \\
\hline Known thrombophilia & $6(2.7 \%)$ \\
\hline Surgery in previous 4 weeks & $1(0.5 \%)$ \\
\hline Significant injury in the previous 4 weeks & $1(0.5 \%)$ \\
\hline History of thrombosis & $17(7.8 \%)$ \\
\hline First trimester & $20(9.1 \%)$ \\
\hline Second trimester & $81(37.0 \%)$ \\
\hline Third trimester & $118(53.9 \%)$ \\
\hline Multiple pregnancy & $11(5.0 \%)$ \\
\hline Long-haul travel during pregnancy & $20(9.1 \%)$ \\
\hline Three or more days of immobility/bed rest & $13(5.9 \%)$ \\
\hline
\end{tabular}

imaging required). The YEARS algorithm was positive in seven women and negative in five. The Geneva score was high in two women, intermediate in nine women and low in one. D-dimer was positive in eight women using the conventional threshold and positive in six women using a threshold double the conventional threshold.

Table 5 also shows whether the women with PE received anticoagulation prior to blood sampling, as this may interfere with D-dimer measurement. Ten women received anticoagulation, ranging from 0 to 16 hours before sampling. One woman was recorded as having no anticoagulation prior to blood sampling but elsewhere was recorded as commencing thromboprophylaxis 3 months prior to presentation. It is therefore not clear whether her D-dimer result could have been influenced by anticoagulant treatment. One woman had no anticoagulation prior to blood sampling and, although PE was considered the most likely diagnosis and the Geneva score indicated intermediate risk of PE, both strategies indicated no PE on account of her negative D-dimer result $(263 \mathrm{ng} / \mathrm{mL})$.

\section{DISCUSSION}

Main findings

Our findings suggest that PE rule out strategies based on clinical probability assessment and D-dimer do not reliably rule out all PE in pregnant women with suspected PE who would otherwise require imaging. The YEARS/D-dimer strategy would have missed five of the 12 and the Geneva/D-dimer strategy would 
Table 2 Characteristics of the women diagnosed with PE

\begin{tabular}{|c|c|c|c|c|}
\hline Patient no & Maternal age & Gestational age & Imaging method & Imaging report \\
\hline 1 & 35 & $28 / 40$ & CTPA & Segmental PE lower left lobe \\
\hline 2 & 34 & $37 / 40$ & VQ & Isolated wedge shaped perfusion defect in the apical segment of the right lower lobe \\
\hline 3 & 26 & $36 / 40$ & CTPA & Extensive bilateral PE \\
\hline 4 & 33 & $35 / 40$ & CTPA & $\begin{array}{l}\text { On balance of probability non-occlusive filling defect in the left upper lobe segmental } \\
\text { vessel represents PE }\end{array}$ \\
\hline 5 & 26 & $23 / 40$ & VQ & Perfusion defects in both lungs \\
\hline 6 & 33 & $24 / 40$ & VQ & $\begin{array}{l}\text { Extensive reduction of perfusion to the right lung with further segmental areas of } \\
\text { perfusion loss in the left lung. The ventilation scan is almost normal. }\end{array}$ \\
\hline 7 & 26 & $32 / 40$ & US lower limb & $\begin{array}{l}\text { Echogenic thrombus demonstrated within the left common femoral vein, which } \\
\text { extends down to the left popliteal vein. The vein was not patent and incompressible }\end{array}$ \\
\hline 8 & 21 & $29 / 40$ & CTPA & Appearances are highly suspicious for solitary small PE \\
\hline 9 & 33 & $24 / 40$ & VQ & Unmatched perfusion defect in the right mid to lower lung \\
\hline 10 & 32 & $27 / 40$ & VQ & Appearances are in keeping with a right acute PE \\
\hline 11 & 25 & $15 / 40$ & VQ & $\begin{array}{l}\text { The segmental perfusion defect in the right lung posteriorly is mismatched with the } \\
\text { ventilation images. Findings are in line with PE }\end{array}$ \\
\hline 12 & 34 & $13 / 40$ & CTPA & Extensive bilateral pulmonary embolus \\
\hline
\end{tabular}

have missed three of the 12 women diagnosed with PE in the DiPEP study.

These findings appear to be inconsistent with the original studies of these algorithms, which identified no symptomatic VTE on follow-up of 46 women with negative assessment using the Geneva/D-dimer strategy and only one DVT on follow-up of 195 women with negative assessment using the YEARS/D-dimer strategy. This apparent inconsistency may be explained by the different designs of the studies, both of which provide useful information and neither of which should be considered definitive. DiPEP is a diagnostic accuracy study in which strategies are compared with an imaging reference standard. The Righini and van der Pol studies are management studies that estimate the risk of adverse outcome in women who have PE ruled out without imaging. Interpretation requires consideration of the strengths and limitations of both designs, and the consequences of missed PE.

The relatively low prevalence of PE in the Righini and van der Pol study cohorts means that even if the strategies missed a quarter of the cases of PE, this would only result in a few missed cases among those discharged without imaging or treatment. The outcomes of PE without treatment are difficult to estimate but it is conceivable that a small number of missed cases could occur without leading to serious adverse outcome. Similarly, the three and five women with PE in the DiPEP study who were respectively missed by the Geneva/D-dimer and YEARS/D-dimer strategies could have survived without adverse event if they had not been treated. A larger cohort of women receiving no treatment after negative assessment would be required to determine

Table 3 Results of applying the YEARS/D-dimer strategy (N, \%, 95\% Cl)

\begin{tabular}{llcc}
\hline & PE & No PE & Total \\
\hline Strategy & 7 & 116 & 123 \\
positive & 58.3 & 56.0 & 56.2 \\
& 28.6 to 83.5 & 49.0 to 62.9 & 49.3 to 62.8 \\
Strategy & 5 & 91 & 96 \\
negative & 41.7 & 44.0 & 43.8 \\
& 16.5 to 71.4 & 37.1 to 51.0 & 37.2 to 50.7 \\
Total & 12 & 207 & 219 \\
\hline
\end{tabular}

$\mathrm{PE}$, pulmonary embolism. whether missed cases led to an unacceptable rate of adverse outcome.

It is an interesting observation that when using the van der Pol and modified Geneva scores, the five 'missed' PE were reported as small or segmental (see table 2). Moreover, these five had the lowest values of D-dimer. These two findings suggest that the volume of the emboli and/or lung tissue affected was small. Unfortunately, the techniques used to identify the PE cannot accurately assess the size of the thrombus and area of pulmonary damage. These observations tentatively suggest that the two scores do detect large PE. However, ignoring small PE is not a safe strategy as these may be a harbinger of later large PE.

The inconsistency between our study and previous studies is unlikely to be explained by differences in the study populations. The study populations had a similar prevalence of PE. The two previous studies were prospective and our analysis was limited to prospectively recruited pregnant women with suspected PE. The two previous studies both recruited women who would otherwise have received imaging (and the authors caution against extrapolating findings to other, lower risk, women) and our analysis was limited to those who received imaging. The van der Pol study described recruitment as consecutive, while the Righini and DiPEP studies did not. However, the recruitment rates across the studies suggested that the DiPEP population was not a more highly selected population, with 219 women recruited across eleven sites over 19 months, compared with 498 across 18 sites over 56 months recruited by van der Pol et al and 395 across eleven sites over 96 months recruited by Righini et al.

Table 4 Results of applying the Geneva/D-dimer strategy (N, \%, 95\% CI)

\begin{tabular}{llll}
\hline & PE & No PE & Total \\
\hline Strategy & 9 & 164 & 173 \\
positive & 75.0 & 79.2 & 79.0 \\
& 42.8 to 93.3 & 72.9 to 84.4 & 72.9 to 84.1 \\
Strategy & 3 & 43 & 46 \\
negative & 25.0 & 20.8 & 21.0 \\
& 6.7 to 57.2 & 15.6 to 27.1 & 15.9 to 27.1 \\
Total & 12 & 207 & 219 \\
\hline
\end{tabular}

$\mathrm{PE}$, pulmonary embolism. 
Table 5 Application of the rule out strategies to women with PE

\begin{tabular}{|c|c|c|c|c|c|c|c|c|c|}
\hline Patient & Haemoptysis & $\begin{array}{l}\text { Clinical signs } \\
\text { DVT }\end{array}$ & $\begin{array}{l}\text { PE most likely } \\
\text { diagnosis }\end{array}$ & $\begin{array}{l}\text { Geneva } \\
\text { score }\end{array}$ & D-dimer & $\begin{array}{l}\text { Thrombo- } \\
\text { prophylaxis }\end{array}$ & $\begin{array}{l}\text { Anticoagulant (time } \\
\text { before D-dimer) }\end{array}$ & $\begin{array}{l}\text { YEARS/D-dimer } \\
\text { strategy }\end{array}$ & $\begin{array}{l}\text { Geneva/D-dimer } \\
\text { strategy }\end{array}$ \\
\hline 1 & No & Yes & No & 12 & 708 & No & $\begin{array}{l}\text { Dalteparin } \\
7500 \text { IU, } 14 \text { hours }\end{array}$ & PE & PE \\
\hline 2 & No & No & No & 5 & 1469 & No & $\begin{array}{l}\text { Dalteparin } \\
6000 \text { IU, } 2 \text { hour }\end{array}$ & PE & PE \\
\hline 3 & No & No & Yes & 5 & 4802 & Yes & $\begin{array}{l}\text { Tinzaparin } \\
19000 \mathrm{IU}, 16 \text { hours }\end{array}$ & PE & PE \\
\hline 4 & No & No & No & 6 & 261 & Yes & No* & No PE & No PE \\
\hline 5 & No & No & No & 0 & 823 & No & $\begin{array}{l}\text { Tinzaparin } \\
175 \mathrm{u} / \mathrm{kg}, 0 \text { hour }\end{array}$ & PE & PE \\
\hline 6 & No & No & No & 5 & 1444 & No & $\begin{array}{l}\text { Dalteparin } \\
7500 \text { IU, } 4.5 \text { hours }\end{array}$ & PE & PE \\
\hline 7 & No & Yes & Yes & 9 & 2696 & No & $\begin{array}{l}\text { Dalteparin } \\
7500 \text { IU, } 7 \text { hours }\end{array}$ & PE & PE \\
\hline 8 & No & No & No & 5 & 662 & No & $\begin{array}{l}\text { Dalteparin } \\
10000 \text { IU, } 7 \text { hours }\end{array}$ & No PE & PE \\
\hline 9 & No & Yes & No & 15 & 194 & Yes & $\begin{array}{l}\text { Dalteparin } \\
12500 \mathrm{IU}, 2 \text { hours }\end{array}$ & No PE & PE \\
\hline 10 & Yes & No & No & 5 & 265 & Yes & $\begin{array}{l}\text { Enoxaparin } \\
80 \mathrm{mg}, 6.5 \text { hours }\end{array}$ & No PE & No PE \\
\hline 11 & No & No & Yes & 5 & 263 & No & No & No PE & No PE \\
\hline 12 & No & No & Yes & 5 & 4329 & No & $\begin{array}{l}\text { Tinzaparin } \\
18000 \text { IU, 13.5 hour }\end{array}$ & $P E$ & PE \\
\hline
\end{tabular}

${ }^{*}$ Recorded as receiving no anticoagulation before blood sampling, but also recorded as commencing tinazaparin 15000 IU daily for thromboprophylaxis 3 months prior to presentation.

DVT, deep vein thrombosis ; PE, pulmonary embolism.

The DiPEP study had limitations that may explain the inconsistency with previous studies. Most women in the DiPEP study received anticoagulation prior to blood sampling. This reflects adherence to UK guidance ${ }^{7}$ that recommends immediate interim parenteral anticoagulant therapy if PE is suspected and imaging cannot be carried out immediately. The process of informing participants and acquiring consent in the DiPEP study meant that blood sampling could not be undertaken immediately. A pooled analysis by Couturaud et $a l^{8}$ estimated that 24 hours after starting heparin therapy D-dimer levels have decreased by $25 \%$ in patients with acute VTE, while a more recent analysis by Baker and Keeling 9 reported a mean decrease of $16 \% 12$ hours after administration of low-molecular-weight heparin. This may have reduced the sensitivity of the strategies but the estimated reductions in D-dimer levels are insufficient to account for the false negative D-dimer results reported in table 4 and would not explain the false negative result in the patient who did not receive anticoagulation prior to blood sampling.

Another limitation of the DiPEP study is that the strategies were applied in theory but not in practice, and the assessment of PE probability was determined according to the documented diagnostic impression. This may be a source of bias if clinicians did not look for or record features such as unilateral lower limb pain, or if interpretation of the documented diagnostic impression is inaccurate. Furthermore, if clinicians are risk-averse, they may deviate from diagnostic protocols in a way that enhances sensitivity at the expense of specificity. For example, many clinicians would not be reassured by a negative D-dimer in the presence of a history of haemoptysis (patient 10) and would over-ride the diagnostic strategy and arrange imaging for PE in this case. The van der Pol and Righini studies have the advantage of showing what clinicians actually do in practice but may be undermined if clinicians exclude eligible patients from the study when they decide to over-ride the strategy.
The recruitment rates in these three studies raise concerns about whether the benefits of using clinical probability assessment and D-dimer to rule out PE are worth the risks. The recruitment rates suggest that less than five women per site per year would avoid imaging. The small cost savings and reduction in radiation risk associated with avoiding a small number of scans per year do not seem to justify the potentially catastrophic consequences of missing PE. For the fetus, the increased risk of childhood cancer to the age of 15 is $0.0006 \%$, while for a mother with a typical background risk of developing breast cancer of $0.1 \%$ in the following 10 years, the absolute risk increase from $10 \mathrm{mGy}$ of radiation is $0.0136 \% .{ }^{10-14}$ Decision analytical modelling undertaken as part of the DiPEP study ${ }^{15}$ suggested that a clinical decision rule to avoid scanning in pregnant and postpartum women with suspected PE would need sensitivity $>97.5 \%$ and specificity $>90 \%$ to be cost-effective compared with a strategy of scanning for all.

\section{CONCLUSION}

We have shown that the combination of clinical probability assessment and D-dimer does not reliably rule out suspected PE in pregnancy. It is uncertain whether PE missed by these strategies lead to clinically important consequences. Recent guidelines from the European Society for Cardiology and European Respiratory Society ${ }^{15}$ suggest using clinical probability and D-dimer to rule out PE in pregnancy, based on the Righini and van der Pol studies. Our findings should be taken into account when considering these guidelines.

Acknowledgements We thank all members of the DiPEP research group.

Contributors SG was the chief investigator for the DiPEP study. This analysis was planned by SG, CN-P, BH and GF. SG undertook the statistical analysis. SG, CN-P, BH and GF contributed to interpretation of the data. SG wrote the initial draft of the 
paper. SG, CN-P, BH and GF contributed to redrafting and approved the final draft of the paper. SG is guarantor for the paper.

Funding The DiPEP study was funded by the UK National Institute for Health Research Health Technology Assessment (HTA) programme (project reference $13 / 21 / 01)$. The funding proposal was submitted in response to a commissioning call that involved patient and public assessment for priority, and was subject to external peer review for quality.

Competing interests The study was funded by the NIHR Health Technology Assessment Programme. SG is deputy director of the HTA Programme and chairs the HTA Commissioning Committee.

Patient and public involvement Patients and/or the public were involved in the design, or conduct, or reporting, or dissemination plans of this research. Refer to the Methods section for further details.

Patient consent for publication Not required.

Ethics approval The DiPEP study was approved by the London Brent Research Ethics Committee (reference 14/LO/1695) on 10 October 2014.

Provenance and peer review Not commissioned; externally peer reviewed.

Data availability statement Data are available on reasonable request from the authors. The data are deidentified participant data, available from the lead author (ORCID 0000-0003-0803-8444), for up to 5 years, under condition that reuse for publication is based on a prospectively registered protocol with a planned analysis.

Open access This is an open access article distributed in accordance with the Creative Commons Attribution 4.0 Unported (CC BY 4.0) license, which permits others to copy, redistribute, remix, transform and build upon this work for any purpose, provided the original work is properly cited, a link to the licence is given, and indication of whether changes were made. See: https://creativecommons.org/ licenses/by/4.0/.

ORCID iDs

Steve Goodacre http://orcid.org/0000-0003-0803-8444

Gordon Fuller http://orcid.org/0000-0001-8532-3500

\section{REFERENCES}

1 van der Pol LM, Tromeur C, Bistervels IM, et al. Pregnancy-adapted years algorithm for diagnosis of suspected pulmonary embolism. N Engl J Med 2019;380:1139-49.
2 Righini M, Robert-Ebadi $\mathrm{H}$, Elias A, et al. Diagnosis of pulmonary embolism during pregnancy: a multicenter prospective management outcome study. Ann Intern Med 2018;169:766-73.

3 Than $M$, Herbert $M$, Flaws $D$, et al. What is an acceptable risk of major adverse cardiac event in chest pain patients soon after discharge from the emergency department?: A clinical survey. Int J Cardiol 2013;166:752-4.

4 Goodacre S, Horspool K, Nelson-Piercy C, et al. The DiPEP study: an observational study of the diagnostic accuracy of clinical assessment, D-dimer and chest $X$-ray for suspected pulmonary embolism in pregnancy and postpartum. BJOG 2019;126:383-92.

5 Hunt BJ, Parmar K, Horspool K, et al. The DiPEP (diagnosis of PE in pregnancy) biomarker study: an observational cohort study augmented with additional cases to determine the diagnostic utility of biomarkers for suspected venous thromboembolism during pregnancy and puerperium. Br J Haematol 2018;180:694-704.

6 Royal College of Obstetricians \& Gynaecologists. Thromboembolic disease in pregnancy and the puerperium: acute management. Green-top guideline no 37b, 2015. Available: https://www.rcog.org.uk/globalassets/documents/guidelines/gtg37b.pdf [Accessed 21 Mar 2016]

7 National Institute for Health and Care Excellence. NICE clinical guideline 144: venous thromboembolic diseases: the management of venous thromboembolic diseases and the role of thrombophilia testing, 2012. Available: http://guidance.nice.org.uk/CG144

8 Couturaud F, Kearon C, Bates SM, et al. Decrease in sensitivity of D-dimer for acute venous thromboembolism after starting anticoagulant therapy. Blood Coagul Fibrinolysis 2002;13:241-6.

9 Baker PM, Keeling DM. A single dose of Low-molecular-weight Heparin (LMWH) invalidates the use of D-dimer as part of a Deep Vein Thrombosis (DVT) diagnostic algorithm. Int J Lab Hematol 2017;39:e17-18.

10 International Commission on Radiological Protection. Pregnancy and medical radiation. Ann ICRP 2000;30:1-43.

11 Hurwitz LM, Reiman RE, Yoshizumi TT, et al. Radiation dose from contemporary cardiothoracic multidetector CT protocols with an anthropomorphic female phantom: implications for cancer induction. Radiology 2007;245:742-50.

12 Remy-Jardin M, Remy J. Spiral CT angiography of the pulmonary circulation. Radiology 1999;212:615-36.

13 Allen C, Demetriades T. Radiation risk overestimated. Radiology 2006;240:613-4.

14 Goodacre S, Horspool K, Shephard N, et al. Selecting pregnant or postpartum women with suspected pulmonary embolism for diagnostic imaging: the DiPEP diagnostic study with decision-analysis modelling. Health Technol Assess 2018;22:1-230.

15 Konstantinides SV, Meyer G, Becattini C, et al. 2019 ESC guidelines for the diagnosis and management of acute pulmonary embolism developed in collaboration with the European respiratory Society (ERS). Eur Heart J 2020;41:543-603. 